$1^{\text {st }}$ International Conference on 3D Materials Science

\title{
3D ANALYSIS OF PHASE SEPARATION IN FERRITIC STAINLESS STEELS
}

\author{
Joakim Odqvist $^{1}$, Jing Zhou ${ }^{1}$, Wei Xiong ${ }^{1}$, Peter Hedström ${ }^{1}$, Mattias Thuvander ${ }^{2}$, Malin Selleby ${ }^{1}$ \\ and John Ågren ${ }^{1}$ \\ ${ }^{1}$ Department of Materials Science and Engineering, KTH Royal Institute of Technology, SE- \\ 10044, Stockholm, Sweden \\ ${ }^{2}$ Department of Applied Physics, Chalmers University of Technology, SE-41296, Göteborg, \\ Sweden
}

Keywords: Phase separation, Atom Probe Tomography, Phase-field modeling

\begin{abstract}
The embrittlement of ferritic stainless steels during low temperature aging is attributed to the phase separation with $\mathrm{Fe}$ and $\mathrm{Cr}$ demixing. The small scale of the decomposed structure with only minor compositional fluctuations and short distances between the enriched and depleted regions has been a challenge for quite some time. A wide selection of experimental and modeling tools have been used to quantify these types of structures. These analyses often focus on rather late stages of decomposition where the mechanical properties are already seriously affected. The recent advance in 3D tools like phase-field and atom probe tomography have created a need for good quantitative procedures of evaluating the structure and also to link results from the continuum approach to the individual atom measurements. This work aims at addressing this need.
\end{abstract}

\section{Introduction}

The phase separation of $\mathrm{Fe}-\mathrm{Cr}$ alloys at low and intermediate temperatures, historically known as the $475^{\circ} \mathrm{C}$ embrittlement, has been studied extensively both experimentally and theoretically, see e.g. [1-4] and the references therein. Due to the small difference in atomic size between Fe and $\mathrm{Cr}$ and the nanoscale structure experimental investigation of phase separation in the early stages using electron microscopy and XRD has been a challenge. However, atom probe tomography (APT) has opened up possibilities to perform atomic-scale studies of short-range ordering and clustering effects [5]. On the theoretical side phase separation has been modeled with the CahnHilliard equation [2,4,6,7] and the Monte Carlo (MC) technique, see e.g. [8]. Both the continuum and atomistic treatments have their advantages and disadvantages: for the Cahn-Hilliard equation additional elements and the effect of stress are easily added, but a quantitative comparison with APT is more challenging. On the other hand, in the MC technique simulation of higher order systems and the influence of stress are not so straightforward to handle while comparison with APT is easier.

In the present work phase separation in one binary $\mathrm{Fe}-\mathrm{Cr}$ alloy is simulated using the CahnHilliard approach coupled with a recently improved thermodynamic description of $\mathrm{Fe}-\mathrm{Cr}$ [3]. A quantitative comparison of the wavelength of compositional fluctuations in simulations and APT is estimated using different methods. 


\section{Atom Probe Tomography (APT)}

Experimental Details

The Fe-Cr alloy was produced by vacuum arc melting and casting of a $600 \mathrm{~g}$ ingot. Before any aging treatments the sample was homogenized at $1100^{\circ} \mathrm{C}$ for $2 \mathrm{~h}$ under argon and subsequently quenched in brine. Aging treatments were performed at $500^{\circ} \mathrm{C}$ for up to $1000 \mathrm{~h}$. Samples for APT were cut into square sectioned rods $20 \times 0.3 \times 0.3 \mathrm{~mm}^{3}$ and subsequently sharp needle-like samples were prepared using the standard two-stage electro-polishing method. A LEAP 3000X $\mathrm{HR}^{\mathrm{TM}}$ (Imago Scientific Instruments, USA) equipped with a reflectron for improved mass resolution was used for all APT measurements. The ion detection efficiency is about $37 \%$ and the experiments were made in voltage pulse mode $(20 \%$ pulse fraction, $200 \mathrm{kHz}$, evaporation rate $1.5 \%$ ) with a specimen temperature of $55 \mathrm{~K}$.

\section{Methods of Analysis}

In the present work focus was on the evaluation of the wavelength of the compositional fluctuations in $\mathrm{Fe}-36$ at. $\% \mathrm{Cr}$ after aging at $500^{\circ} \mathrm{C}$ for $100 \mathrm{~h}$ using three different methods: the autocorrelation function (ACF) [9], fast fourier transform (FFT) and the radial distribution function (RDF) available in the software IVAS 3.4.3.

\section{D Simulation Of Phase Separation In Binary Fe-Cr}

In order to simulate phase separation in binary $\mathrm{Fe}-\mathrm{Cr}$ the ordinary diffusion equation needs to be modified to account for local gradients in composition which enters through the Gibbs energy functional

$$
G=\frac{1}{V_{m}} \int_{V}\left(G_{m}\left(x_{C r}, T\right)+\frac{\kappa^{2}}{2}\left|\nabla x_{C r}\right|^{2}\right) d V
$$

Where the integration is taken over the volume $V$ of interest, $V_{m}$ is the molar volume here assumed to be constant, $G_{m}$ is the molar Gibbs energy, $x_{C r}$ is the mole fraction of $\mathrm{Cr}$ and $\kappa$ are the so-called gradient energy coefficient. The variational derivative of eq. (1) with respect to $x_{C r}$ inserted into the Onsager linear law of irreversible thermodynamic gives us

$$
J_{C r}=-L_{C r C r} \nabla\left(\frac{\delta G}{\delta x_{C r}}\right)=-\frac{L_{C r C r}}{V_{m}} \nabla\left(\frac{\partial G_{m}}{\partial x_{C r}}-\kappa^{2} \nabla^{2} x_{C r}\right)
$$

Where $\mu_{C r}$ and $\mu_{F e}$ are the chemical potentials for $\mathrm{Cr}$ and $\mathrm{Fe}$ respectively. $L_{C r C r}$ denotes the phenomenological coefficient which are related to the atomic mobilities [10]. Eqs. (1) and (2) combined with the continuity equation results in a modified diffusion equation usually called the Cahn-Hilliard equation [6,7].

$$
\frac{1}{V_{m}} \frac{\partial x_{C r}}{\partial t}=-\nabla \cdot J_{C r}
$$


It should be emphasized that no linearization of eq. (3) was performed and the atomic mobilities were allowed to change with composition. Eq. (3) was solved numerically using a semi-implicit Fourier-spectral method [11] on an equidistant grid. The derivatives of the chemical potentials were obtained from Thermo-Calc through a Fortran based programming interface using a recently improved thermodynamic description for the Fe-Cr system [3]. Cahn and Hilliard [6] assumed that the gradient energy coefficient was proportional to the regular solution parameter. This was adopted also in the present work, i.e.

$$
\kappa=\frac{\Omega_{C r F e} l^{2}}{2}
$$

Where $\Omega_{C r F e}$ is the regular solution parameter, taken from [3], $l$ is the interatomic distance set to $0.25 \mathrm{~nm}$. The atomic mobilities were estimated from a fit between composition amplitudes from APT and simulations using a newly developed technique described elsewhere [12]. Simulation results for Fe-36 at.\% $\mathrm{Cr}$ aged at $500^{\circ} \mathrm{C}$ during $0,10,100$ and $180 \mathrm{~h}$ are shown in Fig. 1.

\section{Fe-36 at. \% Cr, $7.04 \times 7.04 \times 7.04 \mathrm{~nm}^{3}$}

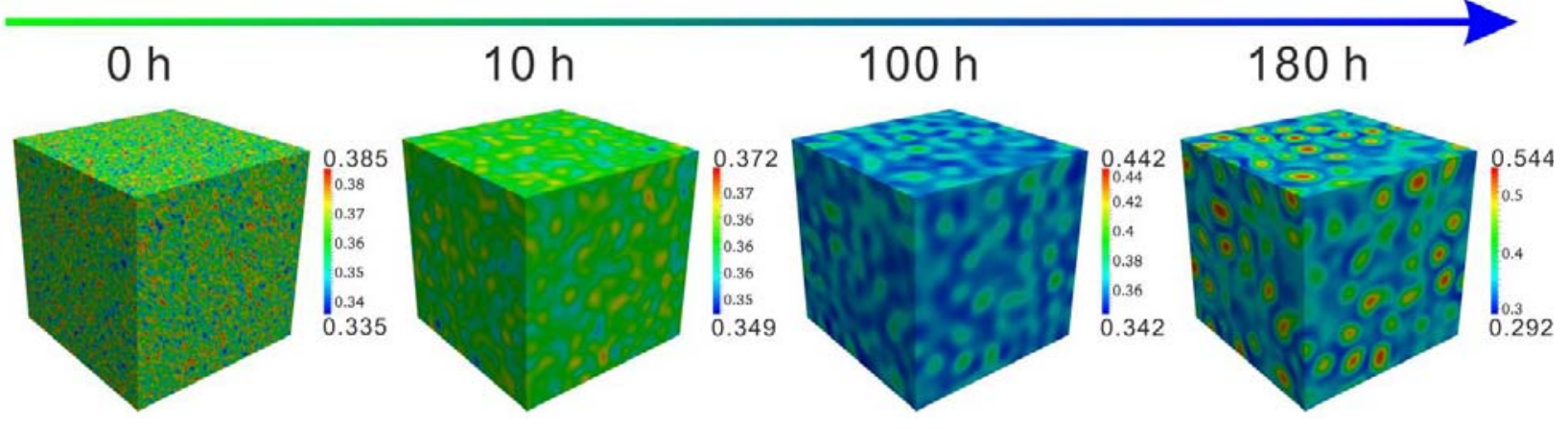

Fig. 1. Simulation of the evolution of phase separation in Fe-36 at. $\% \mathrm{Cr}$ during aging at $500^{\circ} \mathrm{C}$. The red and blue color denotes $\mathrm{Cr}$-rich and Fe-rich areas respectively.

\section{Quantitative Comparison Between Simulation And APT}

The results from evaluation of the wavelength from simulation and APT data using ACF, FFT and RDF are shown in Figs. 2-4. In Fig. 2 the ACF in 1D was used, but it was applied continuously throughout the simulated and experimental volume by stepping in the $\mathrm{y}$ and $\mathrm{z}$ direction while measuring in the x-direction. The results from each step was then added together, hence the smooth curves in Fig. 2. This procedure thus involves the full 3D data and should give a considerably more accurate estimation of the wavelength compared to a 1D ACF in a random direction. In fact, if the 1D ACF was applied to a couple of random directions in the experimental data the estimated wavelengths were in the range 3-5 nm. The first maxima of the ACF indicate the wavelength of the compositional variation [1]: $5 \mathrm{~nm}$ and $1.2 \mathrm{~nm}$ for APT and simulation respectively, see Fig. 2. 


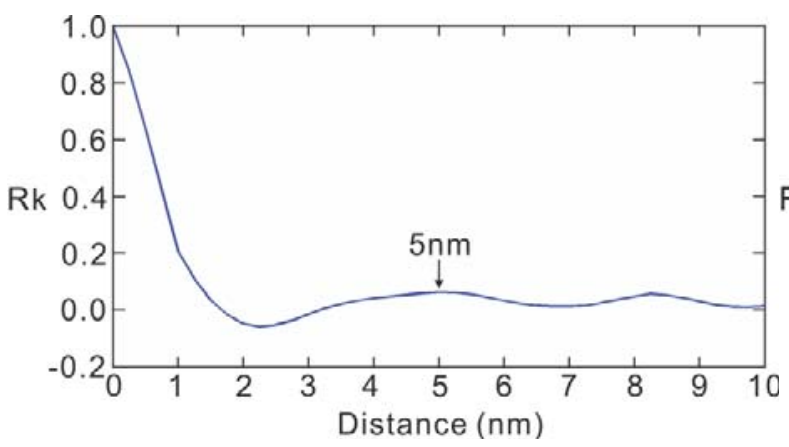

a)

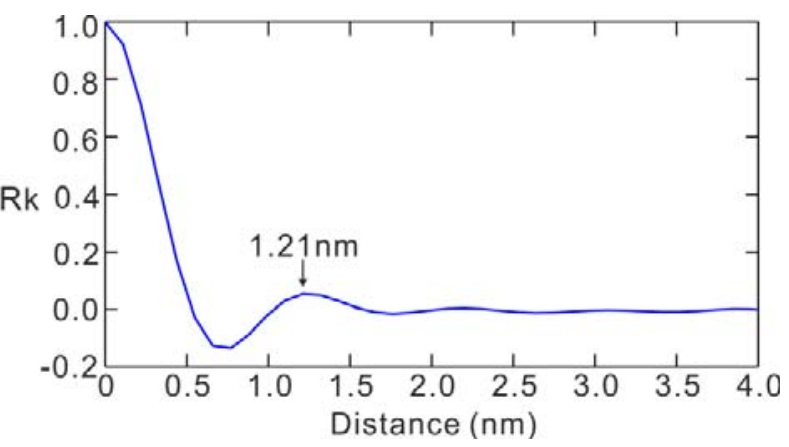

b)

Fig. 2. Results from the autocorrelation function $\left(\mathrm{R}_{\mathrm{k}}\right)$ for a) APT: $\lambda \approx 5 \mathrm{~nm}$ and $\mathrm{b}$ ) Simulation: $\lambda \approx 1.2 \mathrm{~nm}$. After aging Fe-36 at. $\% \mathrm{Cr}$ at $500^{\circ} \mathrm{C}$ for $100 \mathrm{~h}$.

The same procedure as for the ACF was used for the FFT i.e. the FFT in 1D was used repeatedly in the whole volume, see Fig. 3. In order to find the most dominant wave from the spectrum i.e. the fastest growing wave, integration under the different peaks was performed. For APT it was found that the spatial frequency should be between 0.2044 and $0.2166 \mathrm{~nm}^{-1}$, and 0.7032 and $0.7172 \mathrm{~nm}^{-1}$ for the simulation, see the magnified spectrum in Fig. 3. The arithmetic mean for each case was chosen to represent the frequency of the fastest growing wave i.e. $0.21 \mathrm{~nm}^{-1}$ for APT and $0.71 \mathrm{~nm}^{-1}$ for the simulation corresponding to a wavelength of $4.8 \mathrm{~nm}$ and $1.4 \mathrm{~nm}$ respectively.

In Fig. 4 only the RDF for the APT data is shown since no information on the position of individual atoms is available from the simulation. The RDF represents the average radial concentration profile measured from each atom of the species selected; in Fig. 4 the RDF for Cr$\mathrm{Cr}$ is shown. The high values at short distances is an indication that $\mathrm{Cr}$ wants to cluster to $\mathrm{Cr}$ i.e. there is phase separation of $\mathrm{Fe}$ and $\mathrm{Cr}$. If the concentration is normalized with the bulk concentration the RDF will approach unity for a random distribution of $\mathrm{Cr}$ atoms.

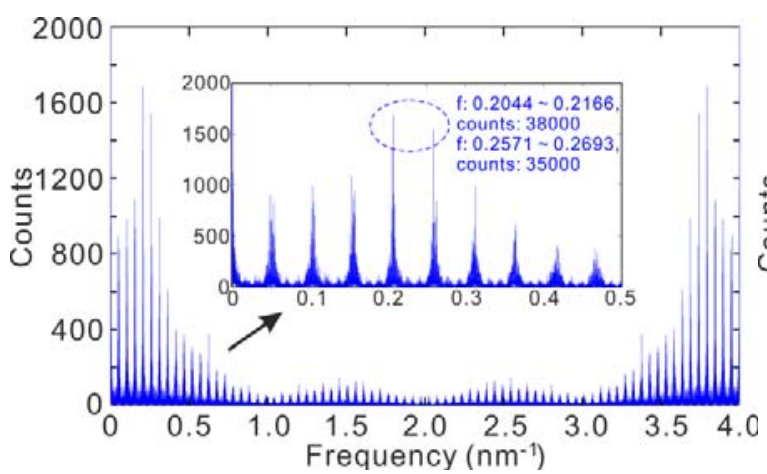

a)

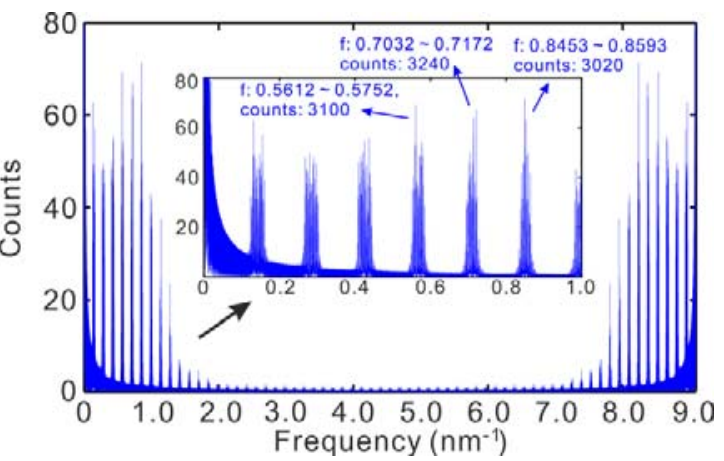

b)

Fig. 3. Results from FFT for a) APT: $\lambda \approx 4.8 \mathrm{~nm}$ and b) Simulation: $\lambda \approx 1.4 \mathrm{~nm}$. After aging Fe-36 at. $\% \mathrm{Cr}$ at $500^{\circ} \mathrm{C}$ for $100 \mathrm{~h}$.

It is interesting to note that the estimated wavelength from the APT data is rather consistent around $5 \mathrm{~nm}$ for all three methods and 1.2-1.4 nm for the simulated data using ACF and FFT. The ACF in 1D applied with the stepping procedure described above seems to perform just as well as the more advanced RDF method, which is believed to be a more accurate method. 
Under the assumption of sinusoidal compositional fluctuations, small in amplitude, Hillert [13] introduced the concept of a critical wavelength $\left(\lambda_{c}\right)$, such as below this wavelength fluctuations would decrease and disappear. For the present case this assumption gives $\lambda_{c}=0.98 \mathrm{~nm}$. Hillert also showed that the wavelength of the fastest growing wave is given by $\lambda_{\max }=\sqrt{ } 2 \lambda_{c}$. Inherent in this result is the assumption of an isotropic material. Inserting the calculated $\lambda_{c}$ gives $\lambda_{\max } \approx 1.4$ $\mathrm{nm}$, in good agreement with the FFT result for the simulation. This good agreement should not come as a surprise since the effects of coherency stresses and anisotropy in elastic properties were not included in the modeling. In reality the fluctuations should be more pronounced in the elastically soft direction. However due to the small misfit between Fe and $\mathrm{Cr}$ this effect should be small.

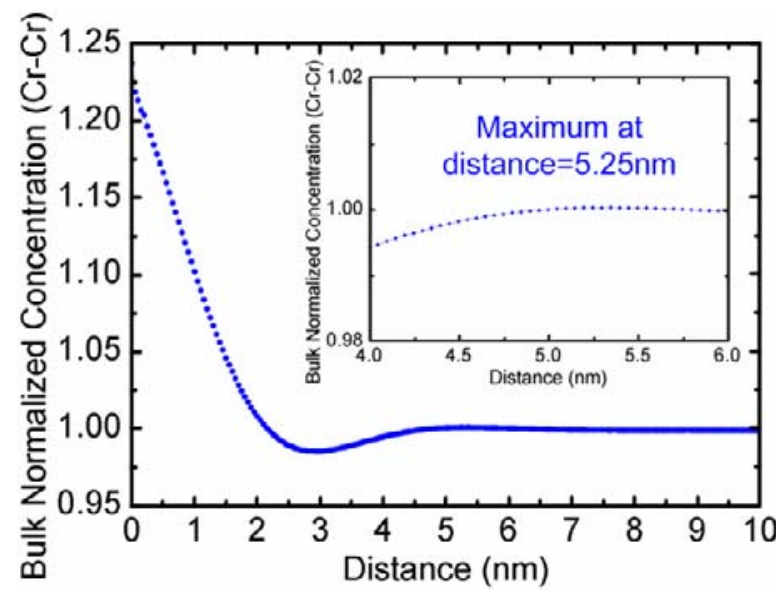

Fig. 4. Results from RDF for APT: $\lambda \approx 5.2 \mathrm{~nm}$. After aging Fe-36 at. $\% \mathrm{Cr}$ at $500^{\circ} \mathrm{C}$ for $100 \mathrm{~h}$.

\section{Concluding Remarks}

In the present work a quantitative comparison of the estimated wavelength of compositional fluctuations during phase separation of a Fe-Cr alloy from APT and simulations has been performed. The large difference in the wavelength estimated from APT and simulation could be due to several reasons. For example, the gradient energy coefficient is assumed to be constant in the simulation, see eq. (4). The gradient energy coefficient is a weight factor that determines how large the energy penalty will be for having a too sharp interface between Fe-rich and Cr-rich regions i.e. the whole gradient term is a measure of the interfacial energy. It is possible that a composition dependent gradient would bring the estimated wavelength from simulations closer to the experimental values. In addition, the Cahn-Hilliard equation is deterministic in the sense that no noise due to thermal fluctuations is accounted for when time $>0$, instead a stochastic variant of the eq. (3) should be solved. All this will be explored in future studies.

\section{Acknowledgements}

This project was supported by the VINNEX centre Hero-m, financed by the Swedish Governmental Agency for Innovation Systems (VINNOVA), Swedish industry and KTH (Royal Institute of Technology) JO and MT also acknowledges the support from the Swedish Research Council (VR, Grant No. 621-2009-5289). 


\section{References}

[1] J. M. Hyde , M. K. Miller, M. G. Hetherington, A. Cerezo, G. D. W. Smith and C. M. Elliott, "Spinodal decomposition in Fe-Cr alloys: experimental study at the atomic level and comparison with computer models - II. Development of domain size and composition amplitude", Acta Metall. Mater., 43 (1995), 3385-3401.

[2] W. Xiong, K. Asp Grönhagen, J. Ågren, M. Selleby, J. Odqvist and Q. Chen, "Investigation of spinodal decomposition in Fe-Cr alloys: Calphad modeling and phase field simulation", Solid State Phenom. 172-174 (2011), 1060-1065.

[3] W. Xiong, P. Hedström, M. Selleby, J. Odqvist, M. Thuvander and Q. Chen, “An improved thermodynamic modeling of the $\mathrm{Fe}-\mathrm{Cr}$ system down to zero Kelvin coupled with key experiments", Calphad, 35 (2011), 355-366.

[4] P. Hedström, S. Bagsheikhi, P. Liu and J. Odqvist, "A phase-field and electron microscopy study of phase separation in Fe-Cr", Mater. Sci. Eng. A, 534 (2012), 552-556.

[5] M.K. Miller and E.A. Kenik, "Atom Probe Tomography: A technique for nanoscale characterization”, Microsc. Microanal., 10 (2004), 336-341.

[6] J.W. Cahn and J. E. Hilliard, "Free energy of a nonuniform system. I. Interfacial energy", $J$. Chem. Phys., 28 (1958), 258-267.

[7] J. W. Cahn, ’On spinodal decomposition”, Acta Met., 9 (1961), 795-801.

[8] C. Pareige, M. Roussel, S. Novy, V. Kuksenko, P. Olsson, C. Domain and P. Pareige, "Kinetic study of phase transformation in a highly concentrated $\mathrm{Fe}-\mathrm{Cr}$ alloy: Monte Carlo simulation versus experiements", Acta Mater., 59 (2011), 2404-2411.

[9] M. Kendall, A. Stuart and J. K. Ord: The Advanced Theory of Statistics $\left(4^{\text {th }}\right.$ Ed. Griffin \& Box, London, 1983).

[10] J.-O Andersson and J. Ågren, "Models for numerical treatment of multicomponent diffusion in simple phases", J. App. Phys., 72 (1992), 1350-1355.

[11] L.Q. Chen and J. Shen, "Applications of semi-implicit Fourier-spectral method to phase field equations", Comp. Phys. Comm., 108 (1998), 147-158.

[12] W. Xiong and J. Ågren, unpublished research, 2012.

[13] M. Hillert, “A solid-solution model for inhomogeneous systems”, Acta Met., 9 (1961), 525535. 